\title{
Comparative evaluation of geological disaster susceptibility using multi-regression methods and spatial accuracy validation
}

\author{
JIANG Weiguo ${ }^{1,2}$, RAO Pingzeng ${ }^{1,2}$, CAO Ran ${ }^{3}$, TANG Zhenghong $^{4}$, CHEN Kun ${ }^{5}$ \\ 1. Academy of Disaster Reduction and Emergency Management, Beijing Normal University, Beijing 100875, \\ China; \\ 2. Key Laboratory of Environmental Change and Natural Disaster, Beijing Normal University, Beijing 100875, \\ China; \\ 3. China Property \& Casualty Reinsurance Company Ltd., Beijing 100033, China; \\ 4. College of Architecture, University of Nebraska-Lincoln, NE 68588, USA; \\ 5. School of Geography and Planning, Ludong University, Yantai 264025, Shandong, China
}

\begin{abstract}
Geological disasters not only cause economic losses and ecological destruction, but also seriously threaten human survival. Selecting an appropriate method to evaluate susceptibility to geological disasters is an important part of geological disaster research. The aims of this study are to explore the accuracy and reliability of multi-regression methods for geological disaster susceptibility evaluation, including Logistic Regression (LR), Spatial Autoregression (SAR), Geographical Weighted Regression (GWR), and Support Vector Regression (SVR), all of which have been widely discussed in the literature. In this study, we selected Yunnan Province of China as the research site and collected data on typical geological disaster events and the associated hazards that occurred within the study area to construct a corresponding index system for geological disaster assessment. Four methods were used to model and evaluate geological disaster susceptibility. The predictive capabilities of the methods were verified using the receiver operating characteristic (ROC) curve and the success rate curve. Lastly, spatial accuracy validation was introduced to improve the results of the evaluation, which was demonstrated by the spatial receiver operating characteristic (SROC) curve and the spatial success rate (SSR) curve. The results suggest that: 1) these methods are all valid with respect to the SROC and SSR curves, and the spatial accuracy validation method improved their modelling results and accuracy, such that the area under the curve (AUC) values of the ROC curves increased by about $3 \%-13 \%$ and the AUC of the success rate curve values increased by $15 \%-20 \% ; 2$ ) the evaluation accuracies of LR, SAR, GWR, and SVR were $0.8325,0.8393,0.8370$ and 0.8539 , which proved the four statistical regression methods all have good evaluation capability for geological disaster susceptibility evaluation and the evaluation results of SVR are more reasonable than others; 3 ) according to the evaluation results of SVR, the central-southern Yunnan Province are the highest sus-
\end{abstract}

Received: 2016-09-08 Accepted: 2016-10-20

Foundation: National Natural Science Foundation of China, No.41571077, No.41171318; The Fundamental Research Funds for the Central Universities

Author: Jiang Weiguo, PhD, E-mail: jiangweiguo@bnu.edu.cn; caoran_1990@163.com 
ceptibility areas and the lowest susceptibility is mainly located in the central and northern parts of the study area.

Keywords: geological disaster susceptibility; multi-regression methods; geographical weighted regression; support vector regression; spatial accuracy validation; Yunnan Province

\section{Introduction}

Typical geological disasters include collapses, landslides, debris flows, flash floods, earthquakes and mixed type geological disasters, etc. (Yi et al., 2012; Samarasundera et al., 2014). In recent decades, geological disasters occurred more frequently because of immoderate use of natural resources by humans. Geological disasters are quite common all over the world, especially in developing countries (Guzzetti et al., 1999; Alcántara-Ayala, 2002; Huang and Cheng, 2013). In fact, nearly every country or region has experienced geological disaster events during the past decades (Metternicht et al., 2005; Samarasundera et al., 2014). Geological disasters threaten the life and property of local people and cause huge damages to the ecological environment, which severely restricts the sustainable development of human society (Uitto and Shaw, 2016). The frequency and intensity of geological disasters intensified rapidly over the most recent decades (Guzzetti et al., 1999). Therefore, it is very meaningful to evaluate geological disaster susceptibility and identify high susceptibility zones for prevention and control of geological disasters.

Geological disaster susceptibility evaluation is the first step to assessing the associated hazards and risks (Varnes, 1984; Trigila et al., 2015). Qualitative and quantitative methods are often used for geological disaster susceptibility evaluation (Wang and Sassa, 2005; Corominas et al., 2014). Because of technical limitations and inaccurate understanding of geological disasters at early stages, researchers often use qualitative assessment methods to evaluate geological disaster susceptibility (Degg, 1992; Zhou et al., 2002). In recent decades, with the progress of science and technology, especially the continuous development of computer technology and geographic information system technology, the quantitative analysis method has gradually become the primary research direction (Bai et al., 2010). There are some commonly used methods, such as the Analytic Hierarchy Process (Nie et al., 2001; Komac, 2006), Fuzzy Comprehensive Evaluation (Jiang et al., 2009), and Logistic Regression (Lee and Pradhan, 2007). Among them, the Analytic Hierarchy Process and Fuzzy Comprehensive Evaluation are heuristic methods that are subjective and empirical with low accuracy. Logistic Regression is a statistical method that collects and regresses historical data to obtain a susceptibility degree index of geological disasters (Ayalew and Yamagishi 2005; Lee and Pradhan, 2007; Li et al., 2015). At present, statistical methods are widely used to evaluate geological disaster susceptibility (Erener and Düzgün, 2010). In addition, with the development of computer technology in recent years, deterministic methods based on the physical mechanisms of objects have been gradually applied to the physical modelling of geological disasters (Pradhan et al., 2016). Two common methods include stability analysis of individual slope formation (Frattini et al., 2004; Frattini et al., 2008) and simulation and analysis of the evolution of single small watershed (Bregoli et al., 2015). Because the parameters are difficult to obtain and the applicability of deterministic methods are limited, so far they have been rarely used in geological disaster susceptibility evaluation (Carrara et al., 2008; Bregoli et al., 2015). 
Statistical methods such as Logistic Regression (LR), Spatial Autoregression (SAR), Geographical Weighted Regression (GWR), and Support Vector Regression (SVR) are usually used to evaluate geological disaster susceptibility, and they are convenient for determining the contribution of various influencing factors to instability (Neuhäuser and Terhorst, 2007; Pourghasemi et al., 2013; Pederson et al., 2015). LR is the most widely used statistical method because of its simplicity and good function, and its effectiveness has been well proven (Ayalew and Yamagishi, 2005; Lee and Sambath, 2006; Lee and Pradhan, 2007; Bai et al., 2010; Ramani et al., 2011; Jiang et al., 2015; Wang et al., 2015). However, there are many factors that LR does not consider that are indispensable, which sometimes causes large errors. Therefore, more and more researchers have adopted an improved statistical regression method or a more comprehensive statistical approach to evaluate geological disaster susceptibility. Currently, the common methods include SAR (Erener and Düzgün, 2011), GWR (Sabokbar et al., 2014), and SVR (Yao et al., 2008; Xu et al., 2012; Pradhan, 2013). SAR is an improved ordinary linear regression technique that recognizes the spatial autocorrelation of the dependent variable by introducing a spatial lag variable and spatial contiguity matrix (Erener and Düzgün, 2010). The traditional logistic regression method does not consider spatial nonstationarity, which is inevitable (Brunsdon et al., 2002; Wu and Zhang, 2013). When spatial nonstationarity is large enough, the error cannot be ignored. In contrast, GWR considers spatial nonstationarity (Fotheringham et al., 1997), and it can be applied well to geological disaster susceptibility evaluation (Sabokbar et al., 2014). SVR applies to small samples and high-dimensional space, thus avoiding over-fitting problems and enabling a strong ability for generalization (Basak et al., 2007). In order to demonstrate the practicality and reliability of SVR, many researchers utilize it for comparison with other traditional methods. Pradhan et al. (2013) contrasted SVR with Decision Trees and neuro-fuzzy models for landslide susceptibility evaluation, and the final result showed that SVR was the best. Besides, there are other commonly used statistical methods, such as frequency ratio model (Lee and Sambath, 2006; Lee and Pradhan, 2007), information quantity model (Tan et al., 2015), weight of evidence (Neuhäuser and Terhorst, 2007), artificial neural network (Xu, 2001; Qiu et al., 2014) etc.

It is difficult to clearly and accurately determine a geological disaster susceptibility value. Davis and Goodrich (1990) considered the accuracy evaluation of the models to be a multi-standard problem. The key is to characterize the model sensitivity, specificity, and accuracy (Melchiorre et al., 2006). At present, commonly used methods include the kappa coefficient, ROC curve (Pourghasemi et al., 2012; Wang et al., 2015), success rate curve (Pradhan, 2013), etc. The kappa coefficient method is simple but not specific that it determines accuracy of the models by calculating kappa values of the modelling results. The ROC curve and success rate curve are simple and intuitive, and both can accurately reflect the specificity and sensitivity of the modelling results with good accuracy validation and wide application to geological disaster susceptibility evaluation (Kavzoglu et al., 2014). In fact, the validation results are usually poor and researchers used to consider the modeling approach as the key factor of them. However, the modelling results are also usually affected by the discrete expression of spatial data, which often causes great deviations in evaluation results (Tang et al., 2013). In this paper, a method called spatial accuracy validation, which can make the modelling values closer to the true values, was introduced to improve the 
situation because of the discrete expression of spatial data (Shekhar et al., 2002). The improved results are then used to verify model accuracy.

Geological disasters are very frequent, and the types of disasters in China are various (Yi et al., 2012; Liu et al., 2012; Li et al., 2013; Cui, 2014), especially southwest region of China (Tang and $\mathrm{Wu}, 1990$ ). Yunnan Province is mountainous and rugged, coupled with frequent heavy rainfall and earthquakes. It is susceptible to erupt geological disasters, such as landslides, flash floods, and debris flows (Liu et al., 1992; Lan et al., 2004; Zhang et al., 2011; Jiang et al., 2016). There are few studies regarding geological disaster susceptibility of the entire Yunnan Province. Mostly, studies targeted partial areas of Yunnan Province and utilized traditional empirical assessment methods (Liu and Lei, 2003; Wu, 2015; Zhuang et al., 2015). In this paper, 500 major geological disasters (including landslides, flash floods, debris flows, etc.) that occurred in Yunnan Province from 2000 to 2014 were selected, and related predisposing factors and potential formation conditions were collected.

Therefore, the objective of this paper is to use multi-regression methods (LR, SAR, GWR, SVR) to evaluate the geological disaster susceptibility of the study area. The spatial receiver operating characteristic (SROC) curve and the spatial success rate (SSR) curve are applied to verify and compare the accuracy of these methods. The susceptibility results could provide evidence-based guidance towards preventing and responding to geological disasters in the study area.

\section{Study area}

Yunnan Province, which is located in southwest China (Figure 1), consists of 16 prefecturelevel cities. The study area lies between $21.14^{\circ} \mathrm{N}$ and $29.25^{\circ} \mathrm{N}$ and $97.53^{\circ} \mathrm{E}$ and $106.20^{\circ} \mathrm{E}$,

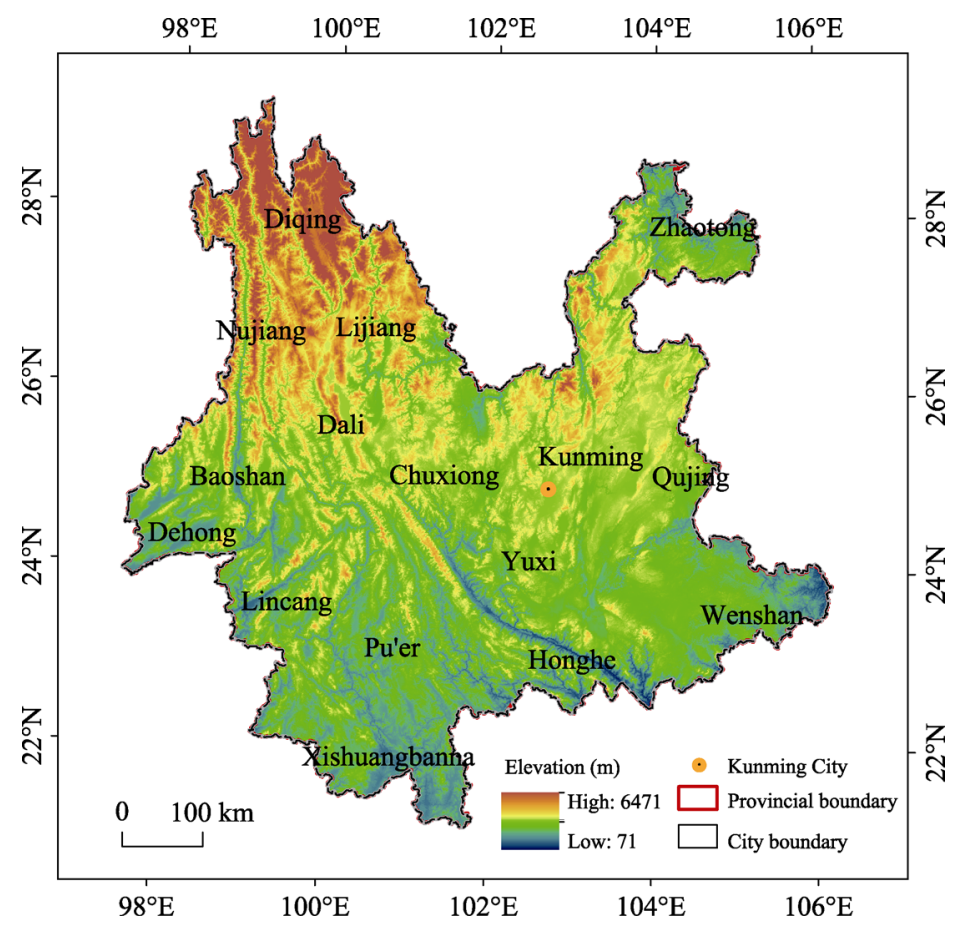

Figure 1 The location of Yunnan Province 
encompassing about $394,000 \mathrm{~km}^{2}$. Northwest of the study area is mountainous area where the altitude is very high; the highest elevation is $6471 \mathrm{~m}$. The central part of the study area belongs to the Yunnan-Guizhou Plateau, with an average elevation of about 2,000 m. Yunnan's mountainous area, which is a typical mountain environment, accounts for $94 \%$ of the province (Liu et al., 2002). Climate change is extremely complex and diverse, and dry and rainy seasons are distinctively obvious. The annual precipitation is more than $1000 \mathrm{~mm}$ inmost of the study area, and the precipitation mainly from June to August with frequent heavy rains. The spatial distribution of precipitation is very uneven, such that it gradually decreases from the northwest to the southeast (Yu et al., 2013).

Earthquakes occur frequently, with approximately 60 instances greater than magnitude 4 per year that lead to great social and economic losses in Yunnan Province (Wen et al., 2008; Yang et al., 2015). The geological structure is extremely complex in the study area. Poor rock slope stability and frequent rain lead to geological disasters such as landslides, flash floods, and debris flows (Zhang et al., 2011; Yang et al., 2015). The annual direct economic loss due to debris flows is more than 8 billion yuan (about 1.3 billion US dollars); furthermore, these disasters resulted in 100 fatalities and a large number of injured people (Liu et al., 2002).

\section{Materials}

\subsection{Disaster samples and non-disaster samples}

The key to susceptibility evaluation is obtaining geological disaster-related spatial attribute data. In this study, data for 500 major disaster events that occurred in Yunnan from 2000 to 2014 were collected, which were mainly downloaded from the National Science \& Technology Infrastructure of China (NSTIC), National Earth System Science Data Sharing Infrastructure (NESSDSI, http://www.geodata.cn) and Chinese Academy of Geological Sciences (CAGS, http://www.geoscience.cn/), including 126 mountain flood events, 91 debris flow events, 271 landslide events, and 12 mixed events evens (Figure 2a).
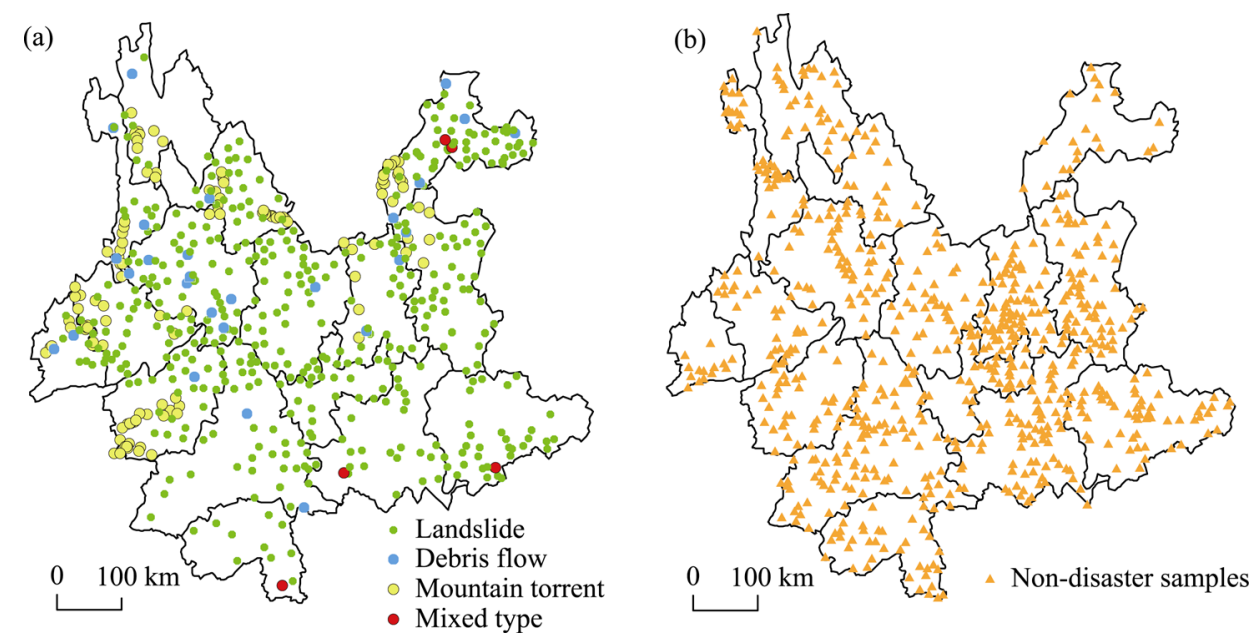

Figure 2 Distribution and types for geological disaster and non-disaster samples 
In addition, non-disaster samples are also important for geological disaster susceptibility evaluation, but usually non-disaster samples are randomly selected within the disaster sample buffer (Ramani et al., 2011). In this study, we argue that geological disaster susceptibility is extremely low in large and medium urban areas. Based on this assumption, we randomly generated non-disaster samples by combining the landslide and debris flow risk zoning maps of Yunnan Province. The interval between non-disaster sampling points is $3 \mathrm{~km}$. Finally, a total of 597 non-point disaster samples were determined according to the above conditions (Figure 2b).

\subsection{Predisposing factors and formation conditions}

It is essential to determine influencing factors that are included as the input variables in the models, including predisposing factors and formation conditions (Figure 3).

Rainfall and earthquakes are direct triggers of geological disasters such as flash floods, debris flows, and landslides in the study area. This paper selected One-hour precipitation, Annual precipitation, and Distance to epicentre as predisposing factors. The precipitation data was obtained from the China Meteorological Administration (CMA), which provided the data from 1692 meteorological stations in and around Yunnan Province. The Inverse Distance Weighted (IDW) spatial interpolation method in ArcGIS was used to interpolate the spatial data of these sites; then One-hour precipitation and Annual precipitation could be calculated using the annual average values of raster data. Seismic data was downloaded from the China Earthquake Data Center (CEDC, http://data.earthquake.cn), with a total of 941 earthquake events in the study area. Distance to epicentre is the Euclidean distance between each pixel and the epicentre that is calculated by the ArcGIS analysis module.

Topography, geological structure, vegetation cover, and river system are the main formation conditions associated with geological disasters. Distance to faults, Fault density, Elevation, Slope, Aspect, Slope position, Lithology, Distance to rivers, and Normalized Difference Vegetation Index (NDVI) were selected as potential indicator factors. Distance to faults and Fault density, which were extracted from 1:2,500,000 geological fault map of China Geological Survey (CGS), reflect features of the geological structure. Distance to faults was obtained using the ArcGIS spatial distance analysis module to calculate the spatial Euclidean distance, and Fault density was calculated using the ArcGIS kernel density analysis method within $10 \mathrm{~km}$ units. Elevation, Slope, Aspect, and Slope position reflect the characteristics of the terrain and were extracted using Shuttle Radar Topography Mission (SRTM) digital elevation data from the National Aeronautics and Space Administration (NASA, http://www. cgiar-csi.org/). River system data was obtained from a 1:250,000 topographic map data of the National Geomatics Center of China (NGCC). Vegetation Index reflects the growing conditions and vegetation cover, and NDVI is the MODIS NDVI product from NASA.

\section{Methods}

\subsection{Evaluation methods of geological disaster susceptibility}

Geological disaster susceptibility evaluation is based on three assumptions: (1) outbreak of geological disasters is mainly related to their influencing factors; (2) historical geological disaster zones can represent very high susceptibility areas; (3) geological disaster susceptibility can be predicted. That is to say, geological disasters susceptibility can be predicted by influencing factors (Lee and Pradhan, 2007; Ramani et al., 2011). Therefore, this study 
adopts the Geological Disasters Susceptibility Index (GDSI) to quantify the degree of geological disaster susceptibility, which can be expressed as a mathematical model. The range of GDSI is $[0,1]$. The specific form of the model is as follows:

(a)

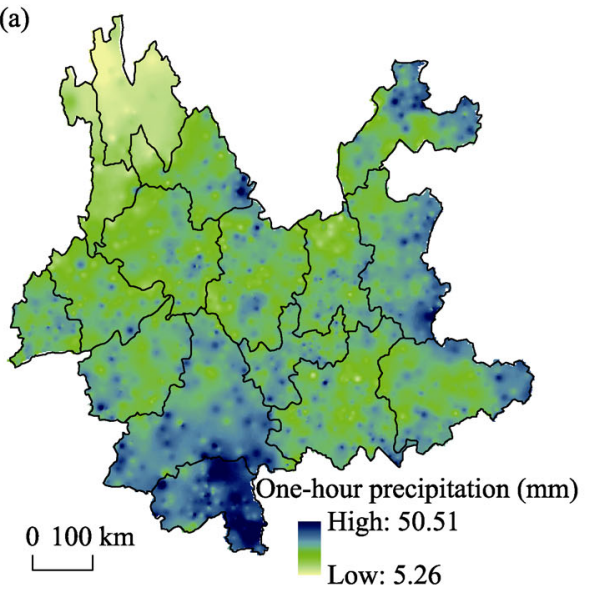

(c)

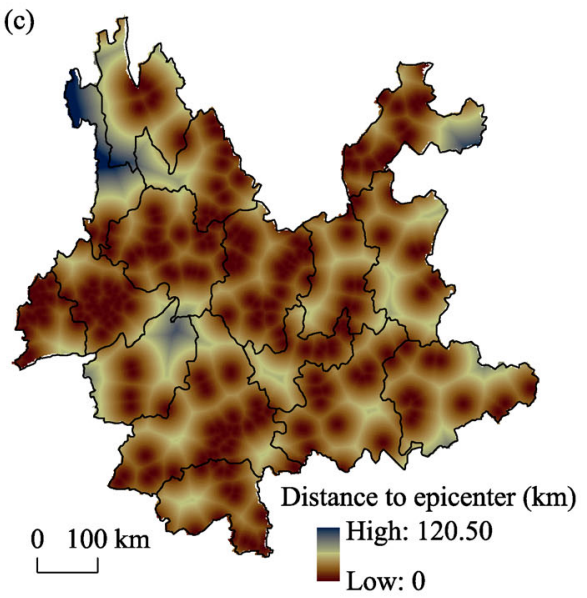

(e)

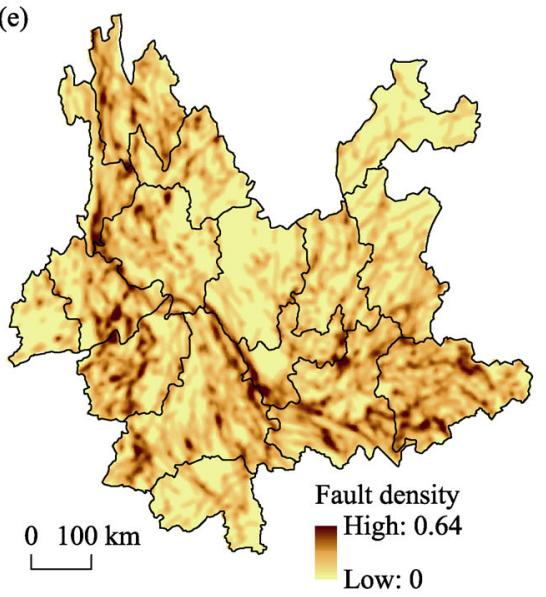

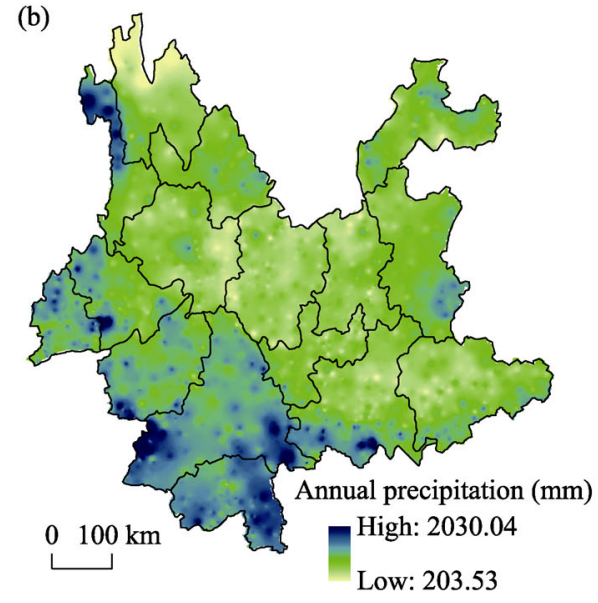

(d)
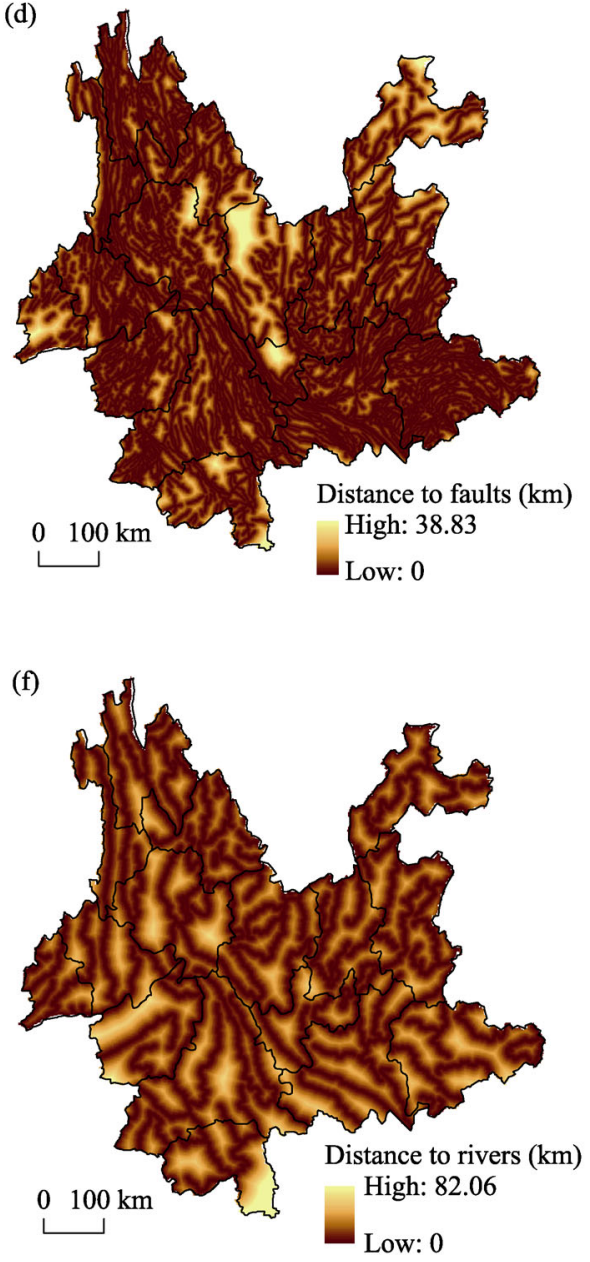

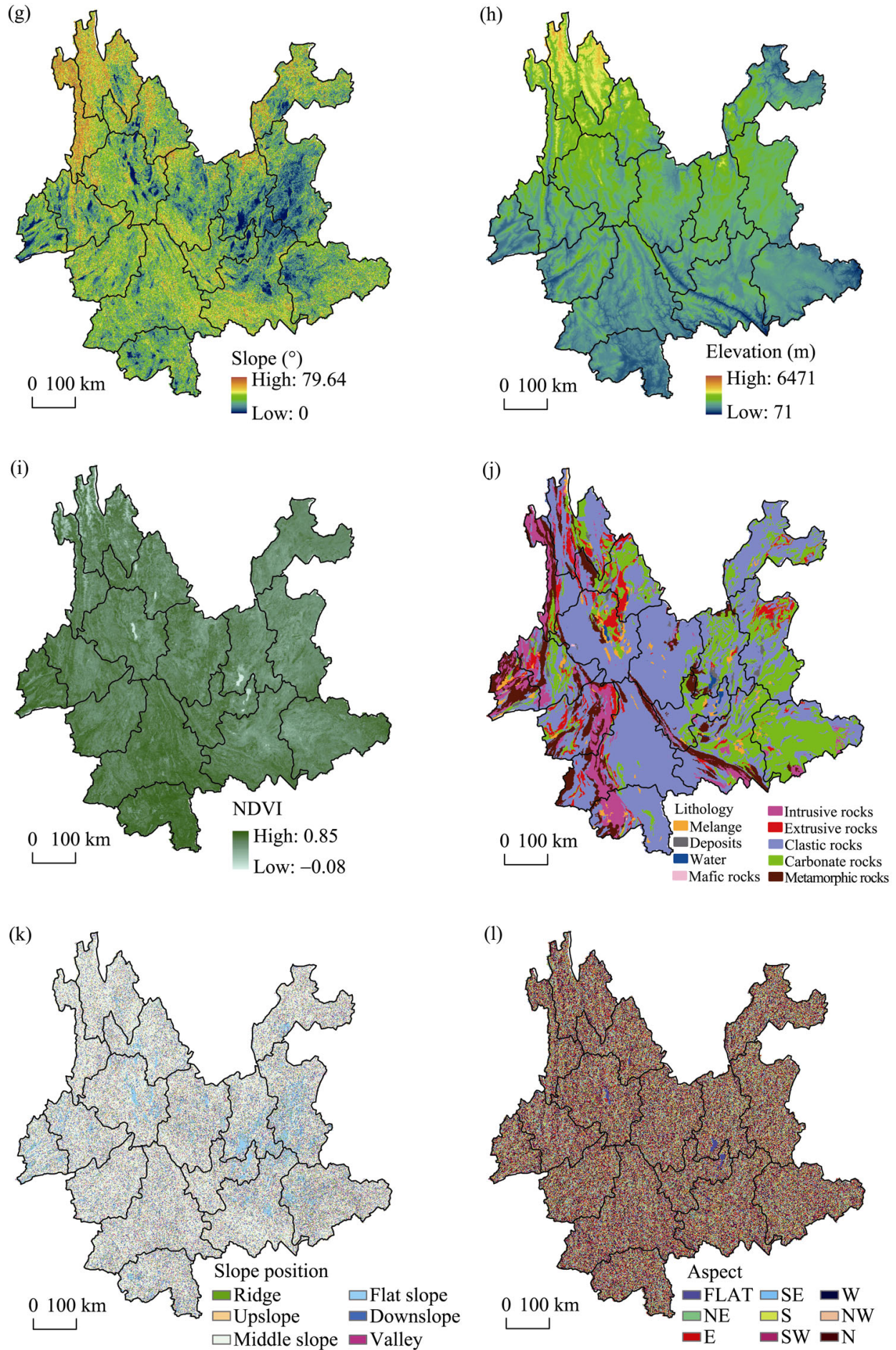

Figure 3 Predisposing factors and formation conditions of geological disaster susceptibility: (a) One-hour precipitation; (b) Annual precipitation; (c) Distance to epicenter; (d) Distance to faults; (e) Fault density; (f) Distance to rivers; (g) Slope; (h) Elevation; (i) NDVI; (j) Lithology; (k) Slope position; (1) Aspect 


$$
G D S I=T\left(f\left(x_{1}, x_{2}, \cdots x_{n}\right)\right)
$$

where $f$ is a spatial math function, $x_{1}, x_{2}, \cdots, x_{n}$ are the susceptibility influencing factors, and $T$ represents the results of linear or non-linear transformation. $f$ is set as LR, SAR, GWR, or SVR.

Importantly, this paper considered 500 geological disasters as positive samples, namely very high susceptibility samples, and their GDSI was set to 1. Similarly, 597 non-geological disasters were considered to be negative samples whose GDSI was set as 0 . The two types of samples were mixed, and $60 \%$ of them were selected as training samples for building and training the evaluation models; the remaining $40 \%$ were used as validating samples for accuracy validation of the modelling results.

\subsubsection{Logistic regression (LR)}

Logistic Regression (LR) is a generalized linear regression method that is widely used to explore the probability of certain events (Wu and Zhang, 2013). Different from ordinary least squares regression, the dependent variables of LR can be categorical variables as well as continuous variables, even if they do not follow the normal distribution. Therefore, it is suitable for geological disaster susceptibility evaluation (Ramani et al., 2011). In the susceptibility evaluation, the dependent variable of LR is GDSI, and the independent variables are influencing factors of geological disasters. GDSI of observed geological disasters samples is either 0 or 1 , representing high susceptibility and low susceptibility, and obey the binomial distribution.

$$
\left\{\begin{array}{l}
P((Y=1 \mid X))=\frac{1}{1+e^{-\varepsilon}} \\
\varepsilon=\beta_{0}+\beta_{1} x_{1}+\beta_{2} x_{2}+\cdots+\beta_{n} x_{n}
\end{array}\right.
$$

where $Y=1$ represents geological disasters, $x_{1}, x_{2}, \cdots, x_{n}$ are known as $n$ influencing factors, and $P(Y=1 \mid X)$ is the probability of a combination of influencing factors, which is also GDSI. $\varepsilon$ is the GDSI linear function, which is assumed to obey a logistic distribution. When $\varepsilon$ approaches negative infinity, $P(Y=1 \mid X)=0$; in contrast, when $\varepsilon$ approaches positive infinity, $P(Y=1 \mid X)=1 . \beta_{0}, \beta_{1}, \beta_{2}, \cdots \beta_{n}$ are the regression coefficients of GDSI, which can be estimated from the observed samples.

\subsubsection{Spatial Autoregression (SAR)}

Spatial Autoregression is a global regression model that introduces spatial lag term and a spatial contiguity matrix (Erener and Düzgün, 2010). The spatial autoregressive linear model expressions are as follows:

$$
\left\{\begin{array}{l}
y=\rho W_{1} y+X \beta+\mu \\
\mu=\lambda W_{2} \mu+\varepsilon \\
\varepsilon \sim N\left(0, \sigma_{\varepsilon}^{2} I_{n}\right)
\end{array}\right.
$$

where $y$ is the independent variable; $\rho$ is the explanatory variable; $W_{1 y}$ is the spatial delay term, where $W_{1}$ is the spatial weights matrix of $y$ and $\rho$ is the regression coefficient of $W_{1 y}$; $W_{2} \mu$ is spatial interference term, where $W_{2}$ is the spatial weights matrix of $\mu$ and $\lambda$ is the regression coefficient of $W_{2} \mu$; and $\varepsilon$ is the residual, which obeys the normal distribution. 


\subsubsection{Geographically Weighted Regression (GWR)}

Geographically Weighted Regression (GWR) is a partial regression model that introduces a spatial non-stationary location and spatial regression coefficients (Fotheringham, 2003). The spatial position is embedded into the regression coefficients, which do not only describe the relationship between explained and explanatory variables but also reflect the spatial variation of their relationship. It is an effective modelling technique used to treat spatial nonstationarity (Su et al., 2012).

$$
y_{i}=\beta_{0}\left(\mu_{i}, v_{i}\right)+\sum_{j=1}^{k} \beta_{j}\left(\mu_{i}, v_{i}\right) x_{i j}+\varepsilon_{i}
$$

where $x_{i j}$ is the independent variable and $y_{i}$ is the dependent variable, $\varepsilon_{i}$ is the residual and $\varepsilon_{i} \sim \mathrm{N}(0, \sigma 2),\left(\mu_{i}, v_{i}\right)$ is the location of $i$, and $\beta_{j}\left(\mu_{i}, v_{i}\right)$ is the regression coefficient of explanatory variables.

GWR uses a locally weighted least squares method to estimate the parameters, and the resulting regression coefficients usually are not constant but variable with respect to changes in spatial position, which reflects the nonstationarity of the spatial relationship (Gao and Li, 2011). The regression parameters of any point can be estimated by the following formula ( $\mathrm{Su}$ et al., 2012):

$$
\hat{\beta}\left(\mu_{i}, v_{i}\right)=\left(X^{T} W\left(\mu_{i}, v_{i}\right) X\right)^{-1} X^{T} W\left(\mu_{i}, v_{i}\right) Y
$$

where $W\left(\mu_{i}, v_{i}\right)$ is a diagonal matrix, and diagonal elements that the distance between point $i$ and point $j$ meet monotonically decreasing (Gao and Li, 2011).

\subsubsection{Support Vector Regression (SVR)}

Support Vector Regression (SVR) based on the statistical learning theory is a nonlinear model for small samples and high-dimensional space (Basak et al., 2007). It converts the inputted space by nonlinear transformation to a high-dimensional feature space and then finds the optimal linear interface in the new space. It avoids the over-fitting problem and has excellent generalization ability.

$\left(x_{i}, y_{i}\right)$ is the training sample set, where $i=1,2, \cdots, l, x \in R, Y \in\{ \pm 1\}$. The regression problem can be converted to the optimal problem, such that the expression is: $\langle W, X\rangle+b=0$

$$
\begin{aligned}
& \min _{w, b, \xi_{i}, \xi_{i}^{*}} \frac{1}{2}\|w\|^{2}+ C \sum_{i=1}^{l}\left(\xi_{i}+\xi_{i}^{*}\right) \\
& \text { subject to }\left\{\begin{array}{l}
y_{i}-\left\langle w, \phi\left(x_{i}\right)\right\rangle-b \leqslant \varepsilon+\xi_{i} \\
\left\langle w, \phi\left(x_{i}\right)\right\rangle+b-y_{i} \leqslant \varepsilon+\xi_{i} \\
\xi_{i}, \xi_{i}^{*} \geqslant 0
\end{array}\right.
\end{aligned}
$$

where $\xi_{i}$ and $\xi_{i}^{*}$ are slack variables, and $\varepsilon$ is a positive constant. When $\left\langle w, \phi\left(x_{i}\right)\right\rangle+b-$ $y_{i} \geqslant \varepsilon$, the value of error is $\left|\left\langle w, \phi\left(x_{i}\right)\right\rangle+b-y_{i}\right|-\varepsilon$; otherwise the error will be discarded.

\subsection{Validation methods of geological disaster susceptibility}

In general, the results of geological disaster susceptibility evaluation need to meet two assumptions: (1) most disaster samples should be located in high susceptibility zones, and most non-disaster samples should be located in low susceptibility zones; (2) the high sus- 
ceptibility zones should occupy a relatively small area and the low susceptibility zones should account for a relatively large area (Can, 2005; Ramani et al., 2011). The receiver operating characteristic (ROC) curve and success rate curve are common methods used for accuracy evaluation of the models. The ROC curve can examine the predictive ability and severability for a model through positive and negative class samples. The success rate curve can examine the ability to predict only disaster samples for a model. Based on the two methods, this study introduced spatial accuracy validation to improve the model evaluation results, and the improved ROC curve and success rate curve should be more reliable.

\subsubsection{ROC curve and success rate curve}

The ROC curve is a method for validating the explanatory capability of the regression models (Pontius, 2001). The contingency table of predicted values and actual values is established by calculating true positive rate (TPR) and false positive rate (FPR) under different susceptibility thresholds of categorized situations. The ROC curve is generated such that TPR is on the ordinate axis and FPR is on the horizontal axis. The area under the curve (AUC) value is the area between the ROC curve and the horizontal axis. The AUC value varies from 0.5 (diagonal line) to 1 , with a higher value indicating a better predictive capability of the model. TPR and FPR are calculated as follows:

$$
T P R=\frac{T P}{T P+F N} \quad F P R=\frac{F P}{F P+T N}
$$

where TP (true positive) is the number of true predictive class samples out of the geological disaster samples; FP (false positive) is the number of false predictive class samples out of the geological disaster samples. TN (true negative) is the number of true predictive class samples out of the non-geological disaster samples; FN (false negative) is the number of false predictive class samples out of the non-geological disaster samples.

Unlike the ROC curve, the success rate curve only considers the predictive capability of the models for the geological disaster samples but disregards the non-geological disaster samples (Pradhan, 2013). The success rate curve is determined by the predictive susceptibility rank (SR) and cumulative percentage $(\mathrm{CP})$ of the corresponding disaster samples, where $\mathrm{SR}$ is the horizontal axis and CP is the vertical axis. SR is often divided into 100 ranks with a descending order of susceptibility.

\subsubsection{Spatial ROC (SROC) curve and spatial success rate (SSR) curve}

Because of the discrete representation of spatial data, the evaluation process often offsets the spatial position. In addition, the spatial data collection and production process also results in accumulation of errors, which yields great uncertainty in the modelling results. Shekhar et al. (2002) considered that the classification for a sample point is correct if the corresponding class appears in or around the pixel of the sample point. That is to say, the predictive values of the observed samples are reasonable (Figure 4). Figure $4 \mathrm{a}$ shows the location of the sample points. Figure $4 \mathrm{~b}$ shows the corresponding pixel location of the actual sample points which is $\mathrm{A}$. $\mathrm{P}$ is the corresponding pixel location of the predicted sample points in Figure 4c. Figure $4 \mathrm{~d}$ shows that $\mathrm{A}$ and $\mathrm{P}$ differ in spatial location, which is considered to be inaccurate for the traditional classification methods, and the accuracy value is 0 . Figure $4 \mathrm{e}$ shows that $\mathrm{P}$ and $\mathrm{A}$ are consistent for a certain space range $(3 \times 3$ pixel areas $)$ because of the existence of a spatial position offset, so the accuracy value is 1 . 
(a)

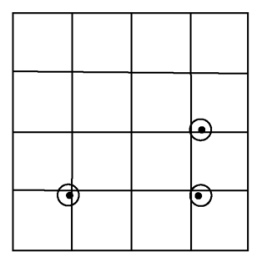

(d)

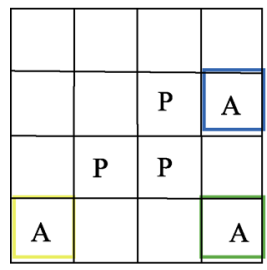

$\mathrm{P} \neq \mathrm{A}$ (b)

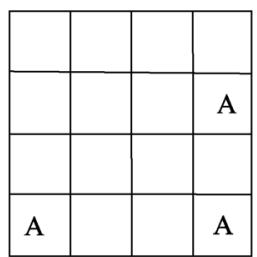

(e)

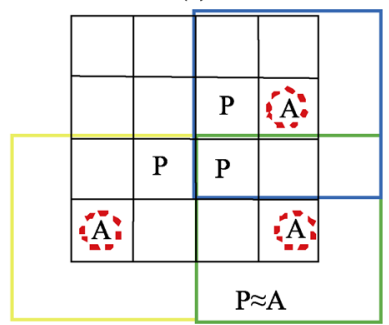

(c)

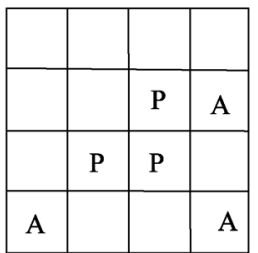

A

Figure 4 Classification accuracy and spatial accuracy (Shekhar, 2002)

Considering that the image resolution is $30 \mathrm{~m}$, this study recounts GDSI of the disaster samples and the non-disaster samples in $3 \times 3$ pixel areas. Finally, we can obtain the spatial ROC (SROC) curve and spatial success rate (SSR) curve.

\subsection{Flowchart}

This study randomly divided disaster samples and non-disaster samples into training samples and validating samples. Training samples were used for modelling, and validating samples were used for validation. The evaluation results of the multi-regression models were evaluated using SROC and SSR. Finally, the results of geological disaster susceptibility were analysed and compared. A flowchart of this process is shown in Figure 5.
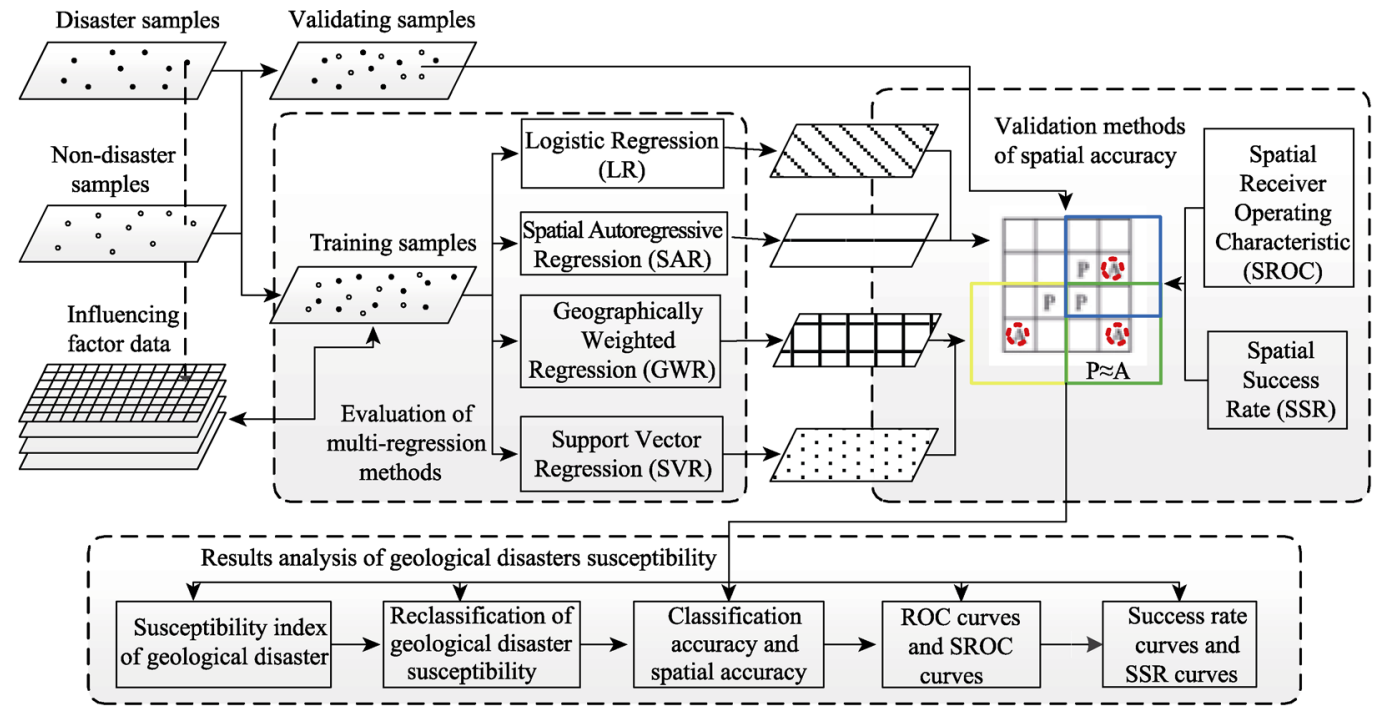

Figure 5 The flowchart of geological disasters susceptibility evaluation 


\section{Results}

In this study, four statistical regression methods were utilized to evaluate geological disaster susceptibility of the study area, and the SROC curve and SSR curve were used to verify and compare the evaluation results of the four methods.

\subsection{Susceptibility index of geological disaster}

The evaluation rsults of the methods are shown in Figure 6. There is a significant difference in the quantity and spatial distribution of the evaluation results for each model. The quantitative feature values of the GDSI, such as the maximum, minimum, mean, and standard deviation are different, as shown in Table 1.

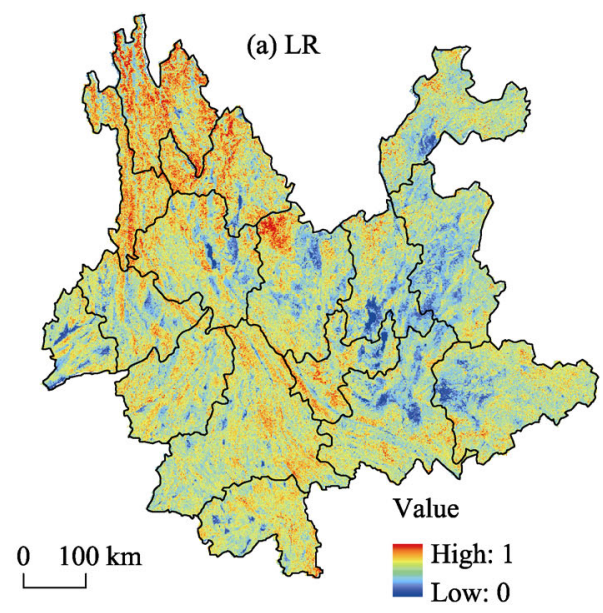

(c) GWR

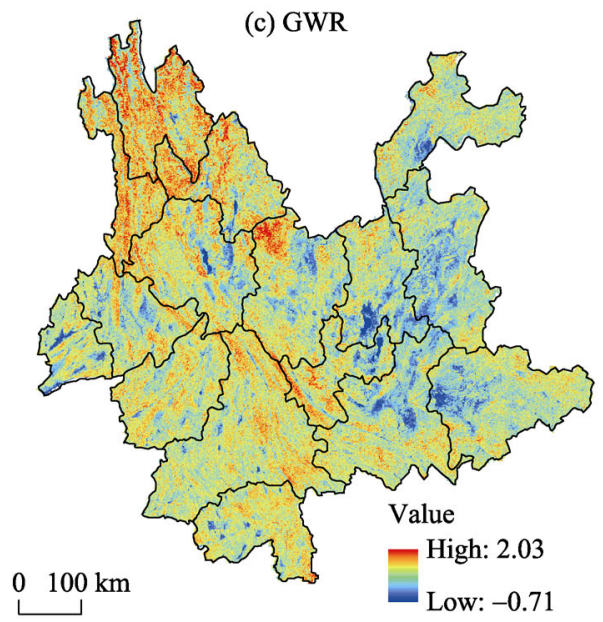

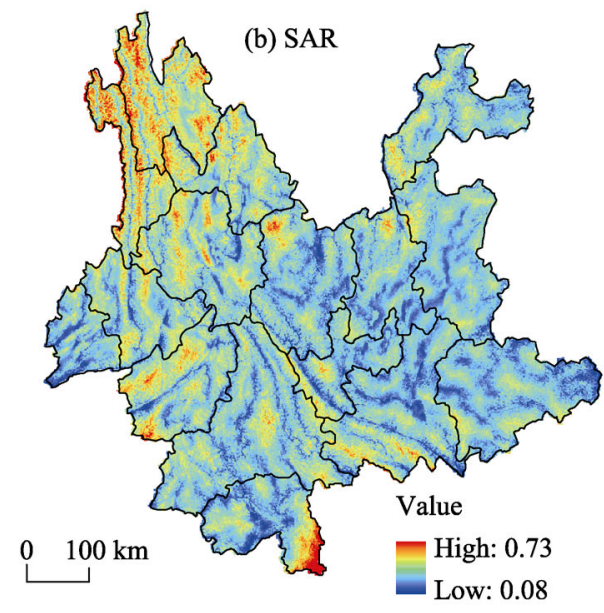

(d) SVR

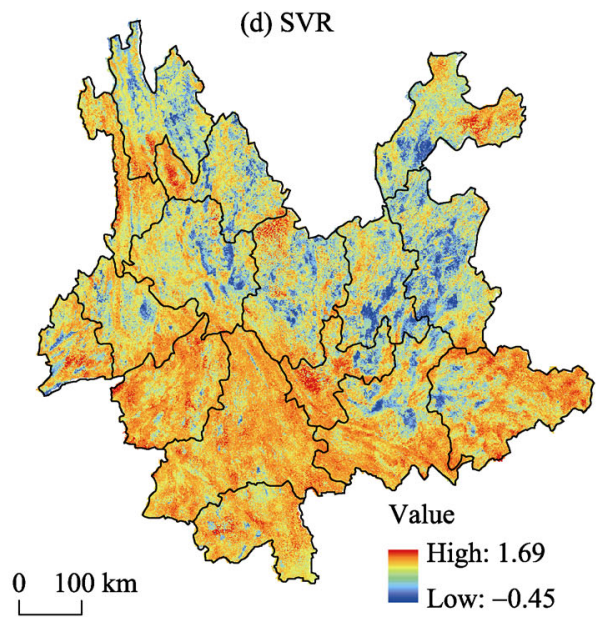

Figure 6 Geological disaster susceptibility index map produced by the multi-regression methods

For LR, the minimum value is 0 and the maximum value is 1 , with a mean of 0.52 and a standard deviation of 0.1 , indicating that the susceptibility level of the study area is relatively uniform. Importantly, for the evaluation results of different models, a maximum value greater than 1 indicates that the predictive capability of the model in high susceptibility areas 
Table 1 Characteristics of GDSI for multi-regression methods

\begin{tabular}{lllll}
\hline & LR & SAR & GWR & SVR \\
\hline Minimum & 0 & 0.077 & -0.71 & -0.45 \\
Maximum & 1 & 0.73 & 2.05 & 1.69 \\
Mean & 0.52 & 0.31 & 0.79 & 0.8 \\
Standard deviation & 0.1 & 0.06 & 0.28 & 0.3 \\
\hline
\end{tabular}

is strong; otherwise, it is weak. Similarly, a minimum value less than 0 indicates that the predictive capability of the model in low susceptibility areas is strong; otherwise, it is weak. Furthermore, the mean value reflects the overall assessment bias of the model. If the mean value is greater than 0.5 , this suggests that the overall evaluation results of the model are relatively large; conversely, they are relatively small.

For the other three models, the predictive capability of SAR in high susceptibility and low susceptibility areas are both weak, and the mean predictive value is relatively small. GWR and SVR are exactly opposite compared to SAR, which indicates that GWR and SVR are better than SAR for geological disaster susceptibility evaluation in the study area.

\subsection{Reclassification of geological disaster susceptibility}

In order to understand geological disaster susceptibility of the study area more completely and clearly, appropriate breakpoints were selected for the cumulative frequency curve to reclassify the susceptibility results. The breakpoints were determined at cumulative frequencies of 30\%, 50\%, 70\%, and 85\% (Hong et al., 2015). There are 5 levels (Very low, Low, Medium, High and Very high levels) for the classification results shown in Figure 7.

Figure 7 shows that the modelling results of LR, SR, and GWR are almost identical, which indicates their principles are relatively similar. The highest susceptibility level is mainly distributed in the northwestern part of the study area, followed by the central and southern regions. Disaster susceptibility is relatively low in the eastern and western parts of the study area. The evaluation results of SVR are clearly different, showing that the northwest of the study area has a low disaster susceptibility but the south has a high disaster susceptibility. It is distinctly different in the northwest of the study area (mainly Diqing city and Lijiang city), as shown in the elliptical area (Figure 7).

\subsection{Classification accuracy and spatial accuracy}

According to the classification criteria, validating samples were selected to count the classification results of the different susceptibility levels (Figure 8), where the proportions of disaster samples reflect the classification levels of different susceptibility classes and the frequency ratio reflects the susceptibility size of different susceptibility classes. For different regression models, the proportions of disaster samples from low to high susceptibility levels were consistent with the selected grade range, namely $30 \%, 20 \%, 20 \%, 15 \%$ and $15 \%$ (section 4.2), which proved the selected breakpoints are reasonable to some extent.

Unfortunately, the results of the frequency ratio are irregular. Theoretically, a higher level of geological disaster susceptibility should correspond to a higher frequency ratio value, but 


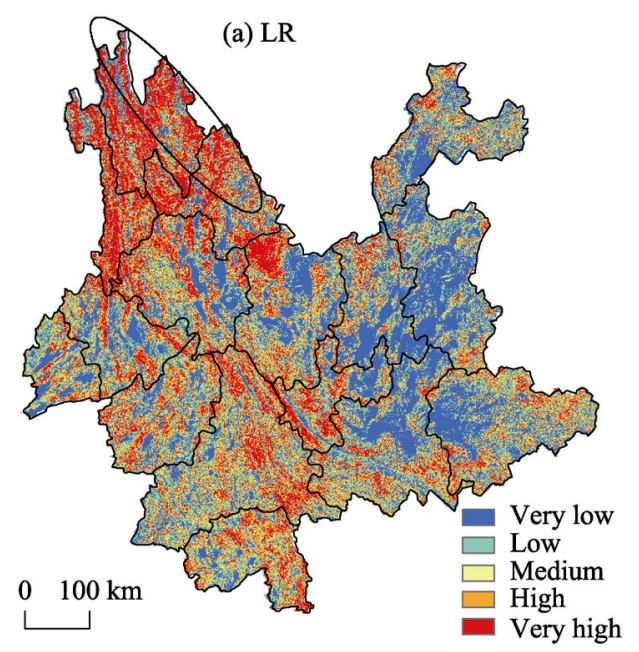

(c) GWR

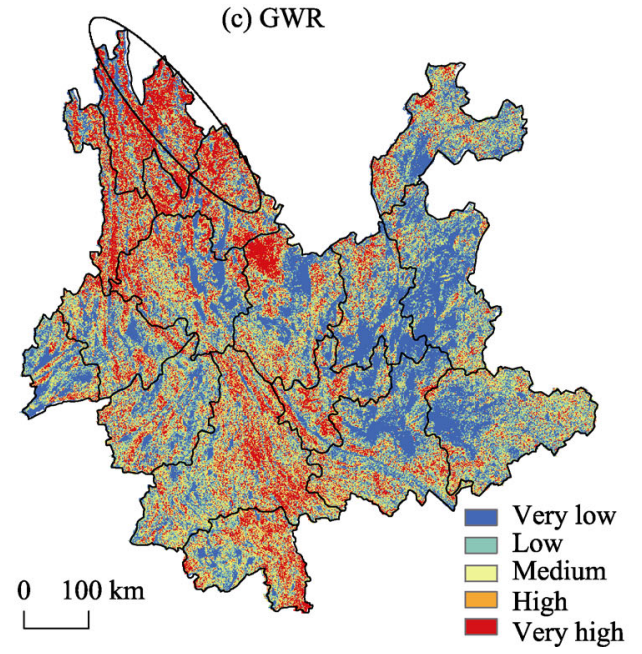

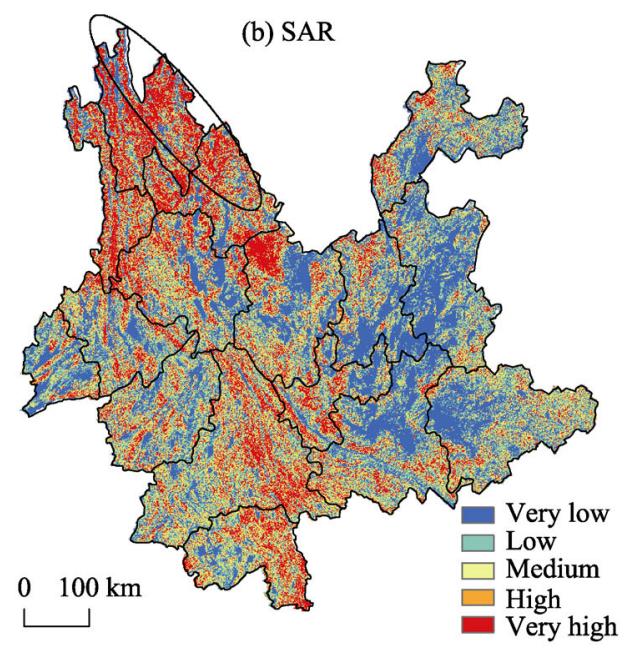

(d) SVR

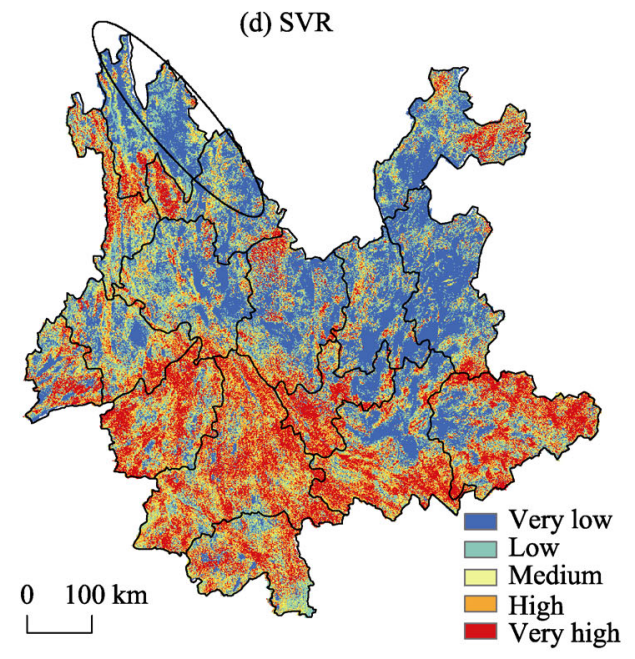

Figure 7 Reclassification of geological disaster susceptibility by the multi-regression methods

the evaluation results of the models obviously do not follow such regularity. For example, the frequency ratio values of LR, ranging from low to high susceptibility levels, are 0.91 , $1.08,0.94,1.13$, and 1.01 , which are all approximately equal to 1 . The other three models are similar to the evaluation results of LR. Such a situation most likely occurs for the following two reasons: (1) the 12 influencing factors that this study selected do not reflect the susceptibility well; or (2) there is not a large error for the assessment results of the models, but discrete expression of the spatial data resulted in offset of the spatial position for disaster sample points, which will affect the susceptibility values of the geological disaster sample points.

Considering that the 12 predisposing factors and formation conditions were selected by combining characteristics of geological disasters in the study area, there is a very low probability that the evaluation results of the four regression methods do not reflect the geological disaster susceptibility of the study area. Therefore, in order to get a better disaster susceptibility distribution map of the study area, this study introduced spatial accuracy validation to 


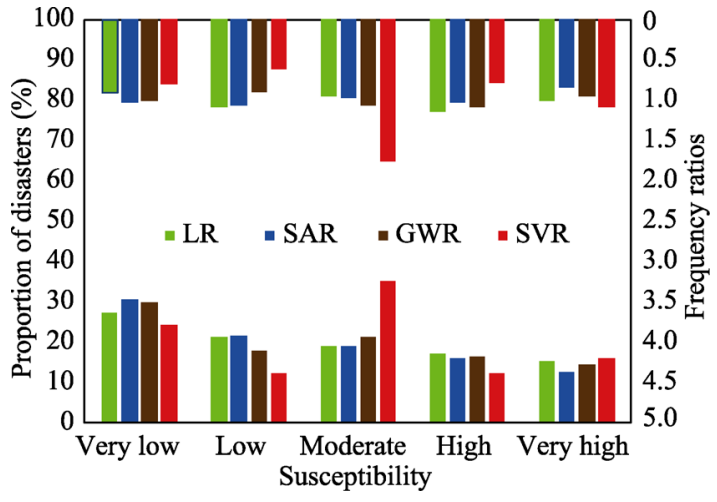

Figure 8 The proportion of disasters and frequency ratios used for validating samples

the neighbourhood of $3 \times 3$ pixels. improve the evaluation results with respect to the second reason mentioned above.

That is, the pixel of a disaster point and the surrounding pixels are all likely to be the true position of the corresponding disaster point, so the highest susceptibility value among them should be considered the susceptibility value of the disaster point. Similarly, for non-disaster sample points, the lowest susceptibility value that appears around them should be considered the susceptibility value of the non-disaster point. In this paper, spatial accuracy is selected as

Figure 9 shows the improved evaluation results of geological disaster susceptibility. It shows that the proportions of disaster samples and the frequency ratio of the four methods are larger significantly in high levels (including 'High' and 'Very high') than before. If disaster sample points distributed in 'Very high' are considered to be reasonable, the simulation results of SVR whose proportions of disasters of 'Very high' is $38.6 \%$ are more reasonable than others. If disaster sample points distributed in 'High' and 'Very high' are considered to be reasonable, the proportions of disasters of LR, SAR, GWR and SVR are $57.6 \%, 55.6 \%$, $57.2 \%$ and $58.0 \%$, respectively, which the simulation results of SVR are also more reasonable than others.

The frequency ratio reflects the degree of the disaster susceptibility, which is closely related to the susceptibility levels. As shown in Figure 9, the evaluation results of LR, with frequency ratios from low to high susceptibility levels being $0.36,0.59,0.99,1.54$, and 2.25, respectively; for SAR, with frequency ratio values of $0.25,0.63,1.24,1.80$ and 1.85 , respectively; for GWR, similarly to the results of LR with frequency ratio values of $0.39,0.60$, $0.97,1.59$ and 2.16, respectively; SVR, with frequency ratio values of $0.41,0.43,1.07,1.24$ and 2.60, respectively. Obviously, the results of the four methods are all good to meet the regularity that the higher susceptibility levels (including 'High' and 'Very high') are corresponding to higher frequency ratio values. Of all the four methods, with the results of proportions of disasters and frequency ratio, the evaluation results of SVR are both more reasonable than others.

Additionally, this study also analysed the distribution of the non-disaster samples at different susceptibility levels and found that most of the non-disaster samples are located in the 'Very low' level, which is consistent with the actual situation. Com-

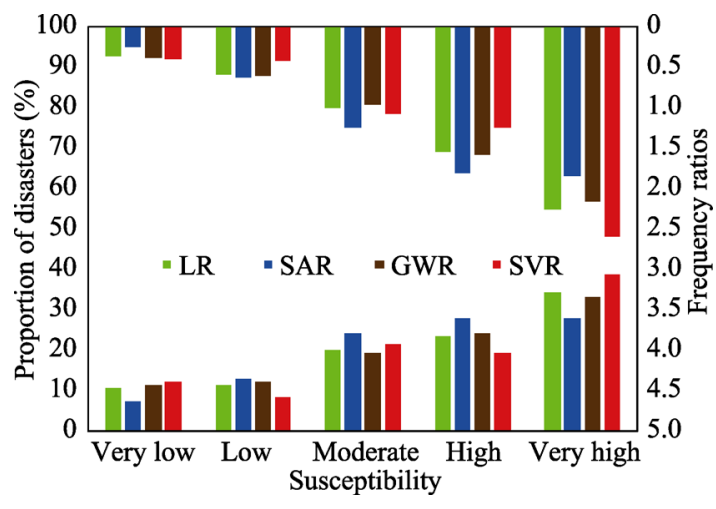

Figure 9 The proportion of disaster samples and frequency ratios introducing spatial accuracy validation 
paring Figures 8 and 9, after introducing spatial accuracy validation methods, the results obtained by the models better reflect the geological disaster susceptibility features of this study area, especially the frequency ratio indicators. In summary, the evaluation results of LR, SAR, GWR and SVR all can reflect geological disaster susceptibility in the study area, and the simulation results of SVR are more reasonable than others.

\subsection{ROC curves and SROC curves}

The ROC curve is used to verify and compare the evaluation results of the models, and the results are shown in Figure 10a. The AUC values of LR, GWR, and SVR are greater than 0.9, indicating that the evaluation effect of each of the three methods is excellent. SAR, with an AUC value of 0.86 , is poorer than other methods, but it is good overall. After introducing the method of spatial accuracy validation, the results are shown in Figure 10b. This shows that the accuracy of the models was improved, and AUC values were all close to 1 after introducing spatial accuracy validation. Compared with the classification accuracy, the effects of the spatial accuracy validation increased by $3 \%-12 \%$, showing that it can better reflect the actual susceptibility results of this study area. It also shows that the four regression methods are all excellent for the geological disaster susceptibility evaluation of this study area.
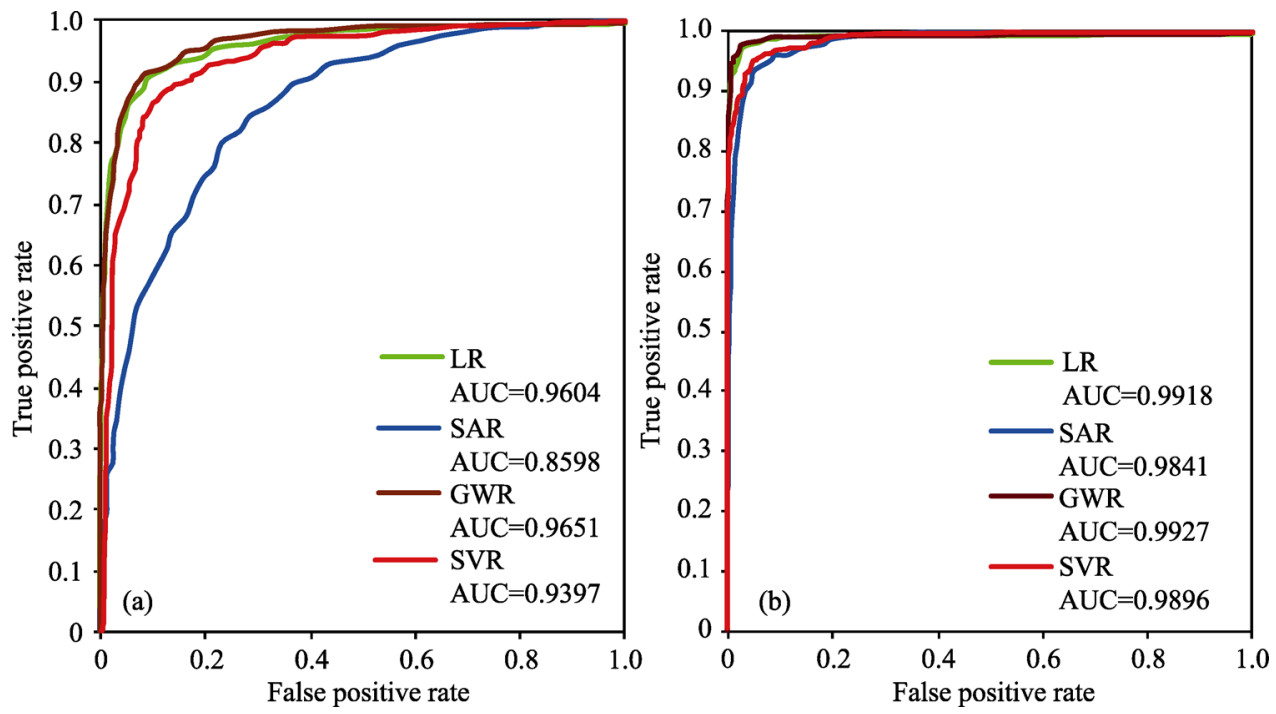

Figure 10 ROC curves of the multi-regression methods: (a) Classification accuracy; (b) Spatial accuracy

\subsection{Success rate curves and SSR curves}

Similarly, the success rate curve is also utilized to verify and compare the evaluation results of the four methods; the results are shown in Figure 11a. The AUC values of the four models are in the vicinity of 0.5 . Generally, when the AUC value is in the range $[0.5,1]$, the simulation results of the model can be considered reliable. For the four models, only the AUC value of SAR does not reach 0.5, indicating that the SAR's simulation result is less reliable. After introducing space accuracy validation, the results are shown in Figure 11b. This figure shows that the accuracy of the models is significantly improved after introducing the method, 
the AUC values of the models were all close to $0.7(>0.5$, indicating that four models have all passed the validation of success rate curve). The method with the highest accuracy was SVR with an AUC value of 0.7183; the lowest was for SAR with an AUC value of 0.6813. After introducing spatial accuracy validation, AUC increased by $15 \%-20 \%$. Compared to the results of the success rate curve, obviously, the results of the SSR curve can better reflect geological disaster susceptibility of this study area.
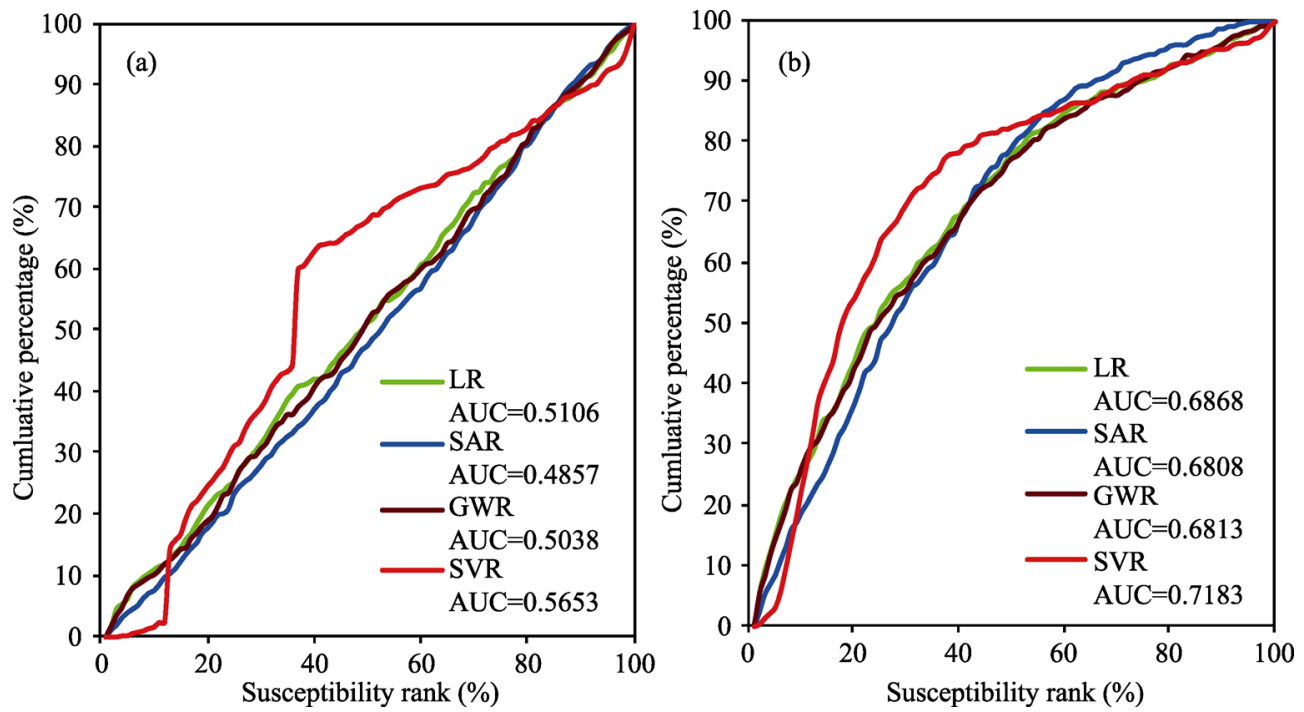

Figure 11 Success rate curves of the multi-regression methods: (a) Classification accuracy; (b) Spatial accuracy

If we think the calculated results of SROC and SSR are reasonable, the AUC values of them are averaged and the AUC values of the four models (LR, SAR, GWR and SVR) are $0.8393,0.8325,0.8370$ and 0.8539 , respectively. Therefore, we can consider the SVR's evaluation effect is the most reasonable, followed by LR, GWR and SAR.

\section{Discussion}

The application of statistical methods for geological disaster susceptibility evaluation is one of the focal points of current geological disaster research. There is huge uncertainty regarding the different geological disaster susceptibility evaluation methods. In order to determine better evaluation methods, this study adopted multi-regression models (including LR, SAR, GWR and SVR) to evaluate the geological disaster susceptibility of the study area. Compared to previous studies, this paper aims to explore the application of statistical regression methods for geological disaster susceptibility evaluation. By contrast, we can determine which methods are superior or not and know the sensitivity and uncertainty of each method. In addition, aiming to address the problem of discrete expressions of spatial data causing space positional displacement, this study introduced spatial accuracy validation to improve the modelling results of these four models. Compared to the previous ROC curve and success rate curve, the effectiveness of improved SROC curve and SSR curve were obviously better. The advantages and disadvantages of the regression methods and validation methods are summarized in below section. 


\subsection{The advantages and disadvantages of multi-regression methods}

Contrasting the four regression methods showed that they have their own distinctive strengths but also many shortcomings. LR is one of the simplest statistical regression methods, whose principle is based on the least squares method. SAR considers spatial autocorrelation, and GWR considers spatial nonstationarity. SVR, unlike LR, SAR and GWR, introduces the vapnik-chervonenkis dimension. Before introducing space accuracy validation, the displayed evaluation results of the four regression methods were poor; afterwards, the evaluation results of all four regression methods reflected the geological disaster susceptibility situation of the study area well, which is demonstrated by the SROC curve (Figure 10) and SSR curve (Figure 11).

For reclassification of geological disaster susceptibility, SAR is lacking in the evaluation of high susceptibility zones, LR, GWR and SVR all yield good simulation results. In terms of the AUC values of the SROC curve and SSR curve, the evaluation results of SVR are the best, followed by LR, GWR and SAR. In addition, compared the evaluation results of the four models, it should be considered why the evaluation results of SVR differed greatly from the evaluation results of the other three methods in northwest of the study area (see Figure 7). In fact, there are little disaster sample points in northwest of the study area. There may be two main reasons which both can cause some deviations for different methods: (1) Predisposing factors and formation conditions selected in this paper are insufficient for geological disaster susceptibility evaluation; or (2) The collection of disaster sample points is very incomplete in northwest of the study area. There are some similar studies on Yunnan Province, and they almost all show that the northwest region witnessed less geological disasters and is not high susceptibility areas (Jiang, 1990; Hu, 2014; Wu, 2015; Jiang 2016). Wu (2015) obtained a similar result with SVR, which shows central and southern parts of the study area are the most susceptibility areas of geological disasters. Based on the analysis of the above, with reference to Figures 2, 6, 7, 8 and 9 and in combination with the disaster sample points and comparison of the evaluation results of the models, the evaluation results of SVR can be considered to be better effect on treating disaster sample points.

The AUC values of LR and GWR are both 0.84 by combining the results of the SROC curves and SSR curves and the evaluation results of LR and GWR are similar, which indicates spatial nonstationarity is weak in the study area. The predictive capability of GWR in high susceptibility areas and low susceptibility areas are both stronger than LR (see Section 4.1), and GWR itself is an improved LR method. Therefore, we can also consider that GWR is better than LR. Unfortunately, compared to the evaluation results of LR, the evaluation results of SAR are relatively poor. It is possible that some regression coefficients deviated or variable factors were selected incompletely.

In this paper, four statistical regression methods are used for geological disaster susceptibility evaluation. In fact, many new similar methods have been widely used in this field, such as multiple logistic regression (Ahmed, 2015), Naïve Bayes classifier (Tsangaratos and Ilia, 2016). In addition, GIS and RS technology is developing rapidly, especially the resolution of image continuously improved. Deterministic methods will be widely applied range in the future, hence the next study is needed to explore the appropriate excitation deterministic method to monitor the susceptibility of various geological disasters. 


\subsection{Advantages and disadvantages of the validation methods}

This paper selected the ROC curve and success rate curve to evaluate the modelling accuracy of the methods. The AUC values of the ROC curves for the models are all approximately 0.9 and nearly 1 for the SROC curves, showing the evaluation accuracy of the models to be excellent. By contrast, the AUC values of success rate curves and SSR curves are about 0.5 and 0.7 , respectively, which are significantly lower than the AUC values of the ROC curves and the SROC curves. Compared to the success rate curve method, the ROC curve considers not only the role of disaster sample points, but also the impact of non-disaster sample points. Thus, the reason that the AUC values of the ROC values and the SROC values are always higher than the AUC values of the success rate curves and the SSR curves is mainly because the non-disaster samples are more reasonable as the assumption than the disaster samples (see Section 4.3). Obviously, the ROC curve method is more persuasive than the success rate curve method.

More importantly, the AUC values of the SROC values and the SSR values are obviously higher than the ROC values and the success rate values. Therefore, we had to pay attention to the spatial accuracy validation method, which was considered in this paper by comparing Figures 10 and 11. It is showed that the spatial accuracy validation method can greatly improve the modelling results, which differs from previous research, and is innovative and valuable for the study of spatially discrete problems in the future, especially for study related to geological disasters.

The disadvantages of the validation methods are summarized in here. Typically, potential geological disasters and formation conditions considered in the study are very uncertain.For example, predisposing factors of geological disasters only considered precipitation and earthquakes in the study. In fact, human activity is also a very important predisposing factor. Negative human activity often intensifies the occurrence of landslides and debris flows, such as unreasonable land use, excessive deforestation, mineral extraction, and even some water conservancy projects. Of course, it is still very difficult to calculate the specific impact of human activity. Besides, this study is mainly based on topographic features of the study area and the degree of difficulty of data acquisition to select influencing factor indicators. Some researchers also consider distance to roads, land cover, and Topographic Wetness Index (TWI), etc. (Regmi et al., 2010; Hong et al., 2015; Trigila et al., 2015). Incompleteness of the selected indicators will cause a certain impact on the results, which should also be considered in future research.

\section{Conclusion}

This paper used different statistical regression methods to evaluate the susceptibility of Yunnan Province, China to typical geological disasters (including landslides, flash floods, debris flows and mixed types). This study introduced the method of spatial accuracy validation to improve the evaluation results which are shown by the SROC curve and SSR curve. Before the improvements, the proportion of disasters and the frequency ratio of each regression model did not correspond to reality. After the improvements, the improved results are consistent with the actual situation, and the proportion of disaster and the frequency ratio increased because the susceptibility levels were increasing. Fortunately, the AUC values of 
the SROC curves increased by 3\%-13\%, and the AUC values of the SSR curves increased by $15 \%-20 \%$, indicating that the space discrete expression causes substantial error for the evaluation of the models.

At last, the evaluation accuracies of LR, SAR, GWR, and SVR were 0.8325, 0.8393, 0.8370 and 0.8539 , respectively, showing that the four statistical regression methods have good evaluation capability for geological disaster susceptibility evaluation. Among them, the evaluation results of SVR are proved to be more reasonable than others. Therefore, the evaluation results of SVR can be considered to be the geological disasters susceptibility mapping of Yunnan. According to the evaluation results of SVR, the central- southern Yunnan Province, including Pu'er, Lincang, Wenshan, Xishuangbanna, southwest of Yuxi and south of Honghe, are the highest susceptibility areas in the province, followed by Nujiang, Zhaotong, Baoshan and Dehong. The lowest susceptibility is mainly located in the central and northern parts of the study area, including Kunming, Qujing, Chuxiong, Dali, Diqing and north of Honghe. The disaster susceptibility research framework in this study can be applied to other study areas.

\section{References}

Ahmed B, 2015. Landslide susceptibility modelling applying user-defined weighting and data-driven statistical techniques in Cox's Bazar Municipality, Bangladesh. Natural Hazards, 79(3): 1707-1737.

Alcántara-Ayala I, 2002. Geomorphology, natural hazards, vulnerability and prevention of natural disasters in developing countries. Geomorphology, 47(2-4): 107-124.

Ayalew L, Yamagishi H, 2005. The application of GIS-based logistic regression for landslide susceptibility mapping in the Kakuda-Yahiko Mountains, Central Japan. Geomorphology, 65(1/2): 15-31.

Bai Shibiao, Wang Jian, Lü Guonian et al., 2010. GIS-based logistic regression for landslide susceptibility mapping of the Zhongxian segment in the Three Gorges area, China. Geomorphology, 115(1/2): 23-31.

Basak D, Pal S, Patranabis D C, 2007. Support vector regression. Neural Information Processing-Letters and Reviews, 11(10): 203-224.

Bregoli F, Medina V, Chevalier G et al., 2014. Debris-flow susceptibility assessment at regional scale: Validation on an alpine environment. Landslides, 12(3): 437-454.

Brunsdon C, Fotheringham A S, Charlton M, 2002. Geographically weighted summary statistics: A framework for localised exploratory data analysis. Computers, Environment and Urban Systems, 26(6): 501-524.

Can T, Nefeslioglu H A, Gokceoglu C et al., 2005. Susceptibility assessments of shallow earth flows triggered by heavy rainfall at three catchments by logistic regression analyses. Geomorphology, 72(1-4): 250-271.

Carrara A, Crosta G, Frattini P, 2008. Comparing models of debris-flow susceptibility in the alpine environment. Geomorphology, 94(3/4): 353-378.

Corominas J, van W C, Frattini P et al., 2013. Recommendations for the quantitative analysis of landslide risk. Bulletin of Engineering Geology and the Environment.

Cui Peng, 2014. Progress and prospects in research on mountain hazards in China. Progress in Geography, 33(2): 145-152. (in Chinese)

Davis P A, Goodrich M T, 1990. A proposed strategy for the validation of ground-water flow and solute transport models. Sandia National Labs., Albuquerque, NM (USA).

Degg M, 1992. Natural disasters: Recent trends and future prospects. Geography, 198-209.

Erener A, Düzgün H SB, 2010. Improvement of statistical landslide susceptibility mapping by using spatial and global regression methods in the case of More and Romsdal (Norway). Landslides, 7(1): 55-68.

Erener A, Düzgün H S B, 2011. Landslide susceptibility assessment: What are the effects of mapping unit and mapping method? Environmental Earth Sciences, 66(3): 859-877. 
FotheringhamA S, Brunsdon C, Charlton M, 2003. Geographically Weighted Regression: The Analysis of Spatially Varying Relationships. John Wiley \& Sons.

Fotheringham A S, Charlton M, Brunsdon C, 1997. Measuring spatial variations in relationships with geographically weighted regression. Recent Developments in Spatial Analysis. Springer Berlin Heidelberg, 60-82.

Frattini P, Crosta G, Carrara A et al., 2008. Assessment of rockfall susceptibility by integrating statistical and physically-based approaches. Geomorphology, 94(3/4): 419-437.

Frattini P, Crosta G B, Fusi N et al., 2004. Shallow landslides in pyroclastic soils: A distributed modelling approach for hazard assessment. Engineering Geology, 73(3/4): 277-295.

Gao Jiangbo, Li Shuangcheng, 2011. Detecting spatially non-stationary and scale-dependent relationships between urban landscape fragmentation and related factors using geographically weighted regression. Applied Geography, 31(1): 292-302.

Guzzetti F, Carrara A, Cardinali M et al., 1999. Landslide hazard evaluation: A review of current techniques and their application in a multi-scale study, central Italy. Geomorphology, 31(1): 181-216.

Hong H, Pradhan B, Xu C et al., 2015. Spatial prediction of landslide hazard at the Yihuang area (China) using two-class kernel logistic regression, alternating decision tree and support vector machines. Catena, 133: 266-281.

Hu Juan, Min Ying, Li Huahong et al., 2014. Meteorological early-warning research of mountain torrent and geologic hazard in Yunnan Province. Journal of Catastrophology, 29(1): 62-66. (in Chinese)

Huang Yu, Cheng Hualin, 2013. The impact of climate change on coastal geological disasters in southeastern China. Natural Hazards, 65(1): 377-390.

Jiang Chaosong, 1990. Geological hazards in Yunnan Province. Journal of Catastrophology, (4): 42-48. (in Chinese)

Jiang Weiguo, Chen Zheng, Lei Xuan et al., 2015. Simulating urban land use change by incorporating an autologistic regression model into a CLUE-S model. Journal of Geographical Sciences, 25(7): 836-850.

Jiang Weiguo, Deng Lei, Chen Luyao et al., 2009. Risk assessment and validation of flood disaster based on fuzzy mathematics. Progress in Natural Science, 19(10): 1419-1425.

Jiang Weiguo, Deng Yue, Tang Zhenghong et al., 2016. Adaptive capacity of mountainous rural communities under restructuring to geological disasters: The case of Yunnan Province. Journal of Rural Studies, 47: $622-629$.

Kavzoglu T, Sahin E K, Colkesen I, 2013. Landslide susceptibility mapping using GIS-based multi-criteria decision analysis, support vector machines, and logistic regression. Landslides, 11(3): 425-439.

Komac M, 2006. A landslide susceptibility model using the analytical hierarchy process method and multivariate statistics in perialpine Slovenia. Geomorphology, 74(1): 17-28.

Lan H X, Zhou C H, Wang L J et al., 2004. Landslide hazard spatial analysis and prediction using GIS in the Xiaojiang watershed, Yunnan, China. Engineering Geology, 76(1/2): 109-128.

Lee S, Pradhan B, 2007. Landslide hazard mapping at Selangor, Malaysia using frequency ratio and logistic regression models. Landslides, 4(1): 33-41.

Lee S, Sambath T, 2006. Landslide susceptibility mapping in the Damrei Romel area, Cambodia using frequency ratio and logistic regression models. Environmental Geology, 50(6): 847-855.

Li Mingze, Lv Jun, Chen Xin et al., 2015. Provincial evaluation of vulnerability to geological disaster in China and its influencing factors: A three-stage DEA-based analysis. Natural Hazards, 79(3): 1649-1662.

Li Xue, Liu Xiaoli, Li Jinggang et al., 2013. Factor analysis of earthquake-induced geological disasters of the M7.0 Lushan earthquake in China. Geodesy and Geodynamics, 4(2): 22-29.

Liu X, Yue Z Q, Tham L G et al., 2002. Empirical assessment of debris flow risk on a regional scale in Yunnan province, southwestern China. Environ. Manage., 30(2): 249-264.

Liu Xilin, Lei Junzhong, 2003. A method for assessing regional debris flow risk: An application in Zhaotong of Yunnan province (SW China). Geomorphology, 52(3/4): 181-191.

Liu Yang, Liu Ronggao, Ge Quansheng, 2010. Evaluating the vegetation destruction and recovery of Wenchuan earthquake using MODIS data. Natural Hazards, 54(3): 851-862.

Melchiorre C, Matteucci M, Azzoni A et al., 2008. Artificial neural networks and cluster analysis in landslide 
susceptibility zonation. Geomorphology, 94(3): 379-400.

Metternicht G, Hurni L, Gogu R, 2005. Remote sensing of landslides: An analysis of the potential contribution to geo-spatial systems for hazard assessment in mountainous environments. Remote Sensing of Environment, 98(2): 284-303.

Neuhäuser B, Terhorst B, 2007. Landslide susceptibility assessment using "weights-of-evidence" applied to a study area at the Jurassic escarpment (SW-Germany). Geomorphology, 86(1/2): 12-24.

Nie H, Diao S, Liu J et al., 2001. The application of remote sensing technique and AHP-fuzzy method in comprehensive analysis and assessment for regional stability of Chongqing City, China. Paper presented at the 22nd Asian Conference on Remote Sensing, 5: 9.

Pederson C A, Santi P M, Pyles D R, 2015. Relating the compensational stacking of debris-flow fans to characteristics of their underlying stratigraphy: Implications for geologic hazard assessment and mitigation. Geomorphology, 248: 47-56.

Pontius R G, Schneider L C, 2001. Land-cover change model validation by an ROC method for the Ipswich watershed, Massachusetts, USA. Agriculture, Ecosystems \& Environment, 85(1): 239-248.

Pourghasemi H R, Moradi H R, Fatemi A S M, 2013. Landslide susceptibility mapping by binary logistic regression, analytical hierarchy process, and statistical index models and assessment of their performances. Natural Hazards, 69(1): 749-779.

Pradhan A M S, Kim Y T, 2016. Evaluation of a combined spatial multi-criteria evaluation model and deterministic model for landslide susceptibility mapping. Catena, 140: 125-139.

Pradhan B, 2013. A comparative study on the predictive ability of the decision tree, support vector machine and neuro-fuzzy models in landslide susceptibility mapping using GIS. Computers \& Geosciences, 51: 350-365.

Qiu Haijun, Cao Mingming, Liu Wen et al., 2014. The susceptibility assessment of landslide and its calibration of the models based on three different models. Scientia Geographica Sinica, 34(1): 110-115. (in Chinese)

Ramani S E, Pitchaimani K, Gnanamanickam V R, 2011. GIS based landslide susceptibility mapping of Tevankarai Ar sub-watershed, Kodaikkanal, India using binary logistic regression analysis. Journal of Mountain Science, 8(4): 505-517.

Regmi N R, Giardino J R, Vitek J D, 2010. Modeling susceptibility to landslides using the weight of evidence approach: Western Colorado, USA. Geomorphology, 115(1/2): 172-187.

Sabokbar H F, Roodposhti M S, Tazik E, 2014. Landslide susceptibility mapping using geographically-weighted principal component analysis. Geomorphology, 226: 15-24.

Samarasundera E, Hansell A, Leibovici D et al., 2014. Geological hazards: From early warning systems to public health toolkits. Health Place, 30: 116-119.

Shekhar S, Schrater P R, Vatsavai R R et al., 2002. Spatial contextual classification and prediction models for mining geospatial data. IEEE Transactions on Multimedia, 4(2): 174-188.

Su Shiliang, Xiao Rui, Zhang Yuan, 2012. Multi-scale analysis of spatially varying relationships between agricultural landscape patterns and urbanization using geographically weighted regression. Applied Geography, 32(2): 360-375.

Tan Yumin, Guo Dong, Bai Bingxin et al., 2015. Geological hazard risk assessment based on information quantity model in Fuling District, Chongqing City, China. Journal of Geo-Information Science, 17(12): 1554-1562. (in Chinese)

Tang Bangxing, Wu Jishan, 1990. Mountain natural hazards dominated (mainly debris flow) and their prevention. Journal of Geographical Sciences, (2): 202-209. (in Chinese)

Tang Y, Atkinson P M, Wardrop N A et al., 2013. Multiple-point geostatistical simulation for post-processing a remotely sensed land cover classification. Spatial Statistics, 5: 69-84.

Trigila A, Iadanza C, Esposito C et al., 2015. Comparison of logistic regression and random forests techniques for shallow landslide susceptibility assessment in Giampilieri (NE Sicily, Italy). Geomorphology, 249: 119-136.

Tsangaratos P, Ilia I, 2016. Comparison of a logistic regression and Naïve Bayes classifier in landslide susceptibility assessments: The influence of models complexity and training dataset size. Catena, 145: 164-179.

Uitto J I, Shaw R, 2016. Sustainable development and disaster risk reduction: Introduction. In: Sustainable De- 
velopment and Disaster Risk Reduction. Springer Japan, 1-12.

Varnes D J, 1984. Landslide hazard zonation: A review of principles and practice. Natural Hazard, 3: 63.

Wang H B, Sassa K, 2005. Comparative evaluation of landslide susceptibility in Minamata area, Japan. Environmental Geology, 47(7): 956-966.

Wang L, Guo M, Sawada K et al., 2015. Landslide susceptibility mapping in Mizunami City, Japan: A comparison between logistic regression, bivariate statistical analysis and multivariate adaptive regression spline models. Catena, 135: 271-282.

Wen Xueze, Ma Shengli, Xu Xiwei et al., 2008. Historical pattern and behavior of earthquake ruptures along the eastern boundary of the Sichuan-Yunnan faulted-block, southwestern China. Physics of the Earth and Planetary Interiors, 168(1/2): 16-36.

$\mathrm{Wu}$ Wei, Zhang Lianjun, 2013. Comparison of spatial and non-spatial logistic regression models for modeling the occurrence of cloud cover in north-eastern Puerto Rico. Applied Geography, 37: 52-62.

Wu Zizi, 2015. Analysis of cause and study on liability assessment of landslide and debris flow hazards in Yunnan province. Beijing: China University of Geosciences. (in Chinese)

$\mathrm{Xu}$ Chong, Dai Fuchu, Xu Xiwei et al., 2012. GIS-based support vector machine modeling of earthquake-triggered landslide susceptibility in the Jianjiang River watershed, China. Geomorphology, 145/146: $70-80$.

$\mathrm{Xu}$ Zengwang, 2001. GIS and ANN model for landslide susceptibility mapping. Journal of Geographical Sciences, 11(3): 374-381.

Yang Wen, Liu Jie, Cheng Jia et al., 2015. The impact of the 24 March 2011 Myanmar earthquake (MS 7.2) on seismic structure of the Yunnan region. Tectonophysics, 649: 165-175.

Yao X, Tham L G, Dai F C, 2008. Landslide susceptibility mapping based on support vector machine: A case study on natural slopes of Hong Kong, China. Geomorphology, 101(4): 572-582.

Yi Lixin, Ge Lingling, Zhao Dong et al., 2012. An analysis on disasters management system in China. Natural Hazards, 60(2): 295-309.

Yu Wenjin, Shao Mingyang, Ren Manliang et al., 2013. Analysis on spatial and temporal characteristics drought of Yunnan Province. Acta Ecologica Sinica, 33(6): 317-324.

Zhang Yongshuang, Zhao Xitao, Lan Hengxing et al., 2011. A Pleistocene landslide-dammed lake, Jinsha River, Yunnan, China. Quaternary International, 233(1): 72-80.

Zhou Jinxing, Wang Lixian, Xie Baoyuan et al., 2002. A study on the early-warning technique concerning debris flow disasters. Journal of Geographical Sciences, 12(3): 363-370.

Zhuang Jianqi, Cui Peng, Wang Gonghui et al., 2015. Rainfall thresholds for the occurrence of debris flows in the Jiangjia Gully, Yunnan Province, China. Engineering Geology, 195: 335-346. 